\title{
ELEMENTOS-TRAÇO E SÓDIO EM SUCO DE UVA: ASPECTOS NUTRICIONAIS E TOXICOLÓGICOS
}

\author{
Roberta Amorim de Assis e Ivo Lewin Küchler* \\ Departamento de Química Analítica, Universidade Federal Fluminense, Alameda Barros Terra, s/n, 24020-141 Niterói - RJ, \\ Brasil \\ Norbert Miekeley e Carmem Lúcia Porto da Silveira \\ Departamento de Química, Pontifícia Universidade Católica do Rio de Janeiro, R. Marquês de São Vicente, 225, 22451-900 \\ Rio de Janeiro - RJ, Brasil
}

Recebido em 10/7/07; aceito em 20/6/08; publicado na web em 10/11/08

\begin{abstract}
TRACE ELEMENTS AND SODIUM IN GRAPE JUICE: NUTRITIONAL AND TOXICOLOGICAL ASPECTS. Eight trace elements were determined in 20 Brazilian brands of grape juice, distributed over the country. Highest measured concentrations (As: 0.016; Cd: 0.010; Cr: 0.060; Cu: 1.28; Ni: 0.032; Pb: 0.016; Sb: 0.0040 and $\mathrm{Zn:} 1.44 \mathrm{mg} \mathrm{L}^{-1}$ ) comply with Brazilian maximal tolerance levels for inorganic contaminants (As: 0.5; Cd: 0.5; Cr: 0.1; Cu: 30; Ni: 3; Pb: 0.4; Sb: 1 and Zn: 25 mg L'-1). Determination of arsenic species has shown inorganic $\mathrm{As}(\mathrm{V})$ as predominant in most samples. Sodium concentrations, nowadays a major public health concern, were also measured, showing an average of $149 \mathrm{mg} \mathrm{L}^{-1}$. Analytical results for this element were much higher than label concentrations, showing the need for better quality control.
\end{abstract}

Keywords: arsenic; sodium; grape juice.

\section{INTRODUÇÃO}

No Brasil, a lista de Limites Máximos de Tolerância (LMT) para contaminantes em suco de fruta ${ }^{1}$ inclui 10 elementos-traço (As: 0,$5 ; \mathrm{Cd}$ : 0,5; Cr: 0,1; Cu: 30; Hg: 0,01; Ni: 3; Pb: 0,4; Sb: 1; Se: 0,05; Sn: 250; e Zn: $25 \mathrm{mg} \mathrm{L}^{-1}$ ). Limites específicos para contaminantes inorgânicos em suco de fruta também podem ser encontrados em outros países, como por exemplo, na Comunidade Européia ${ }^{2}\left(\mathrm{~Pb}: 0,05 \mathrm{mg} \mathrm{kg}^{-1}\right)$, e na Índia (As: 0,2; Cu: 5,0; Pb: 1,0; Sn: 250 e Zn: 5,0 mg kg-1 ). Entre os diversos sucos comercializados no Brasil, o suco de uva encontra-se numa posição de destaque, tanto pela dimensão do seu consumo per capita (0,56 L/ano), como pelo gradual aumento de consumo que tem apresentado nos últimos anos. ${ }^{4}$ Por isso, diversos trabalhos recentes têm abordado a determinação de elementos-traço em suco de uva no Brasil. Por exemplo, Morgano et al..$^{5}$ determinaram cobre, zinco, e outros elementos, em diversos sucos de frutas. Nas duas amostras de suco de uva analisadas, foram encontrados teores de 3,94 e 3,80 mg $\mathrm{L}^{-1}$ de cobre, e 0,56 e $0,61 \mathrm{mg} \mathrm{L}^{-1}$ de zinco. Estas concentrações de cobre, embora abaixo do LMT, são muito superiores às encontradas nos outros tipos de sucos, que apresentam valores na faixa de 0,25 a $0,75 \mathrm{mg} \mathrm{L}^{-1}$. Em um estudo realizado no estado do Rio Grande do Sul, Mirlean et al. ${ }^{6}$ determinaram 6 elementos-traço em suco de uva e vinho da região de Bento Gonçalves. Nas 4 amostras de suco analisadas, foram obtidos os seguintes teores médios, em mg L ${ }^{-1}$ : Cd 0,0013; Cr 0,038; $\mathrm{Cu}$ 0,53; Ni 0,019; Pb 0,117 e Zn 0,307. Em média, as concentrações destes elementos são duas a três vezes maiores no suco de uva que nas amostras de vinho analisadas. Para efeito de comparação com outros países, pode-se citar o trabalho de Olalla et al., ${ }^{7}$ que apresenta teores médios de $0,063 \mathrm{mg} \mathrm{L}^{-1}$ de cobre ( $\mathrm{n}^{\circ}$ de amostras, $\mathrm{N}=60$ ), e $0,46 \mathrm{mg}$ $\mathrm{L}^{-1}$ de zinco $(\mathrm{N}=60)$, em sucos de uva da Espanha, sendo esta concentração de cobre o menor valor encontrado na literatura consultada. O Departamento de Agricultura dos Estados Unidos ${ }^{8}$ considera como referências no suco de uva os valores médios de $0,28 \mathrm{mg} \mathrm{kg}^{-1}$ de cobre $(\mathrm{N}=14)$ e $0,50 \mathrm{mg} \mathrm{kg}^{-1}$ de zinco $(\mathrm{N}=4)$.

\footnotetext{
*e-mail: gqaivo@vm.uff.br
}

Atualmente considera-se que não basta conhecer as concentrações totais dos elementos, devido à grande diferença de toxicidade apresentada por algumas espécies de elementos-traço, como ocorre com o arsênio, antimônio, cromo e mercúrio. No arsênio, a toxicidade das espécies obedece à seguinte ordem aproximada: ${ }^{9}$ arsina $\left(\mathrm{AsH}_{3}\right)>$ arsenitos inorgânicos $>$ compostos trivalentes orgânicos $\left(\mathrm{CH}_{3} \mathrm{As}(\mathrm{OH})_{2}\right.$ e $\left.\left(\mathrm{CH}_{3}\right)_{2} \mathrm{AsOH}\right)>$ arsenatos inorgânicos $>$ compostos pentavalentes orgânicos $\left(\mathrm{CH}_{3} \mathrm{AsO}(\mathrm{OH})_{2}\right.$ e $\left(\mathrm{CH}_{3}\right)_{2} \mathrm{AsO}(\mathrm{OH})>$ compostos arsônicos $>$ arsênio elementar. Sabe-se que o consumo de quantidades sub-letais de arsênio inorgânico por períodos prolongados pode provocar alterações na pele e a ocorrência de câncer. Por outro lado, as espécies orgânicas arsenobetaína $\left(\left(\mathrm{CH}_{3}\right)_{3} \mathrm{AsCH}_{2} \mathrm{COOH}^{+}\right)$e arsenocolina $\left(\left(\mathrm{CH}_{3}\right)_{3} \mathrm{AsCH}_{2} \mathrm{CH}_{2} \mathrm{COOH}^{+}\right)$são consideradas atóxicas. Estes fatos justificam que a determinação de arsênio em alimentos não se restrinja ao teor total do elemento, mas inclua a determinação do arsênio(III) inorgânico, que apresenta um risco à saúde muito superior ao das outras espécies.

O sódio é um elemento essencial, que tem como uma das principais funções, juntamente com o potássio e o cloreto, manter um balanço eletrolítico adequado de fluidos no organismo humano. $\mathrm{O}$ excesso de sódio no organismo leva à acumulação de líquido, o que acarreta aumento do volume de sangue, e conseqüentemente, aumento da pressão sangüínea. A pressão elevada sobrecarrega o coração e pode resultar em outros problemas circulatórios. Atualmente o consumo recomendado de sódio para adultos saudáveis, definido como Valor Diário de Referência (VDR), é de $2400 \mathrm{mg},{ }^{10}$ sendo que pessoas idosas ou com doenças crônicas, como hipertensão, diabetes e insuficiência renal, devem consumir quantidades menores. Por estes motivos, apesar do sódio não ser classificado como um contaminante, o consumo de sódio através de alimentos passou a ser encarado como uma séria questão de saúde pública. Conseqüentemente, as normas sanitárias brasileiras ${ }^{10}$ determinam que seja informada nos rótulos dos produtos alimentícios a massa de sódio (em mg) por porção consumida, podendo ser informada como "zero mg de sódio" ou "não contém sódio", quando inferior a $5 \mathrm{mg}$. Nos Estados Unidos, o valor de referência para sódio em suco de uva é $30 \mathrm{mg} \mathrm{kg}^{-1}(\mathrm{~N}=32)$. 
O presente estudo sobre contaminantes em suco de uva se diferencia dos anteriormente realizados no Brasil pelo maior número de amostras coletadas (31), pela variedade de marcas (20), pela abrangência geográfica (incluindo 9 estados, do Rio Grande do Sul até o Ceará) e pelo número de elementos-traço determinados (8). Além disso, com a utilização da técnica de geração do hidreto acoplada à espectrometria de massa (HG-ICP-MS), ${ }^{11}$ foi realizada a determinação das espécies inorgânicas do arsênio. Finalmente, a determinação de sódio foi incluída no estudo, tendo em vista a importância deste elemento no contexto da saúde pública.

\section{PARTE EXPERIMENTAL}

\section{Técnicas analíticas e instrumentos}

Arsênio total foi determinado por espectrometria de massa com fonte de plasma e geração de hidreto (HG-ICP-MS), usando um sistema comercial de análise por injeção em fluxo FIAS 200 (Perkin Elmer, USA), acoplado a um espectrômetro de ICP-MS ELAN 5000 (PerkinElmer SCIEX, USA), operado sob condições padrão (Tabela 1). Este método foi utilizado para evitar a interferência espectral de ${ }^{40} \mathrm{Ar}{ }^{35} \mathrm{Cl}$ sobre ${ }^{75} \mathrm{As}$, causada pelas altas concentrações de cloreto nas amostras.

Tabela 1. Parâmetros instrumentais típicos dos espectrômetros de massas PerkinElmer ELAN 5000 e ELAN 6000 e do espectrômetro de emissão óptica OPTIMA 4300 DV.

\begin{tabular}{|c|c|c|c|}
\hline & $\begin{array}{c}\text { ELAN } \\
5000 \\
\end{array}$ & $\begin{array}{c}\text { ELAN } \\
6000 \\
\end{array}$ & $\begin{array}{l}\text { OPTIMA } \\
4300 \mathrm{DV}\end{array}$ \\
\hline Tipo de nebulizador & crossflow & crossflow & concêntrico \\
\hline $\begin{array}{l}\text { Tipo de câmara } \\
\text { de nebulização }\end{array}$ & Ryton ${ }^{\circledR}$ & Ryton ${ }^{\circledR}$ & $\begin{array}{l}\text { ciclônica, } \\
\text { vidro }\end{array}$ \\
\hline Potência do Plasma(W) & 1200 & 1200 & 1500 \\
\hline Resolução de massa & 0,8 & 0,8 & - \\
\hline Amostrador e skimmer & platina & platina & - \\
\hline $\begin{array}{l}\text { Vazão do argônio } \\
\text { do plasma }\left(\mathrm{L} \mathrm{min}^{-1}\right)\end{array}$ & 15,0 & 15,0 & 15,0 \\
\hline $\begin{array}{l}\text { Vazão de argônio } \\
\left.\text { auxiliar (L min }{ }^{-1}\right)\end{array}$ & 1,0 & 1,0 & 0,5 \\
\hline $\begin{array}{l}\text { Vazão de argônio } \\
\text { do nebulizador }\left(\mathrm{L} \mathrm{min}^{-1}\right)\end{array}$ & 0,9 & 0,9 & 0,6 \\
\hline Sensibilidade para Rh (cps) & $\begin{array}{c}50.000- \\
60.000\end{array}$ & $\begin{array}{c}200.000 \\
-300.000\end{array}$ & - \\
\hline$\%$ de Óxidos $\left(\mathrm{CeO}^{+}\right)$ & $<3 \%$ & $<3 \%$ & - \\
\hline$\%$ íons bivalentes $\left(\mathrm{Ba}^{2+}\right)$ & $<1 \%$ & $<1 \%$ & - \\
\hline $\begin{array}{l}\text { Sinal de Fundo em } \\
\mathrm{m} / \mathrm{z} 220 \text { (cps) }\end{array}$ & 25 & 25 & - \\
\hline Modos de varredura & peak-hop & peak-hop & - \\
\hline $\begin{array}{l}\text { Tempo de permanência } \\
\text { no pico (dwell time })(\mathrm{ms})\end{array}$ & 60 & 130 & - \\
\hline Varreduras por leitura & 2000 & 1 & - \\
\hline Número de replicatas & 1 & 3 & - \\
\hline Comprimento de onda (nm) & & & 267,716 \\
\hline Vista do sistema óptico & - & - & axial \\
\hline
\end{tabular}

Para a determinação de arsênio total, $1 \mathrm{~mL}$ da amostra foi decomposta com uma mistura de $1 \mathrm{~mL}$ de $\mathrm{HNO}_{3} 70 \%$ e $1 \mathrm{~mL}$ de $\mathrm{H}_{2} \mathrm{O}_{2} 30 \%$, em frasco de polipropileno com tampa, aquecido por uma noite em um bloco de aquecimento a $80^{\circ} \mathrm{C}$. Ao final deste procedimento, que visa destruir compostos refratários de arsênio, evaporou-se o excesso de $\mathrm{HNO}_{3}$. A pré-redução do As(V) a As(III) foi realizada com $1 \mathrm{~mL}$ de uma solução contendo $5 \% \mathrm{~m} / \mathrm{v}$ de KI e $5 \% \mathrm{~m} / \mathrm{v}$ de ácido ascórbico, além de $3 \mathrm{~mL}$ de $\mathrm{HCl} 7 \mathrm{~mol} \mathrm{~L}^{-1},{ }^{11}$ mantendo-se a mistura em repouso durante 15 min. $\mathrm{O}$ hidreto foi gerado por reação com $\mathrm{NaBH}_{4} 0,4 \%$ $\mathrm{m} / \mathrm{v}$, e $\mathrm{HNO}_{3} 0,4 \mathrm{~mol} \mathrm{~L}^{-1}$ foi usado como solução carreadora. O sistema de injeção em fluxo estava equipado com uma membrana gás-líquido $(0,2 \mu \mathrm{m}$, Teflon) e uma alça de amostragem de $250 \mu \mathrm{L}$.

Nos estudos de especiação de arsênio, não foi efetuada a oxidação prévia da amostra com $\mathrm{HNO}_{3} \mathrm{e}_{2} \mathrm{O}_{2}$. Para a determinação da soma de $\mathrm{As}(\mathrm{III})$ e As(V), seguiu-se o procedimento descrito acima, a partir da etapa de pré-redução. ${ }^{11} \mathrm{O}$ As(III) foi determinado com o mesmo procedimento, porém sem pré-redução. $\mathrm{O}$ teor de $\mathrm{As}(\mathrm{V})$ foi obtido por diferença entre os dois resultados anteriores.

A espectrometria de massa com fonte de plasma (ICP-MS) foi utilizada para a determinação de antimônio, cádmio, chumbo, cobre, níquel e zinco. Foi empregado um espectrômetro ELAN 6000 (Perkin ElmerSciex, USA), nas condições padrão do instrumento (Tabela 1), com leitura dos seguintes isótopos: $\mathrm{Cd} \mathrm{114,} \mathrm{Cu} 65, \mathrm{Ni} \mathrm{60,} \mathrm{Pb}$ 208, Rh 103, Sb 121, e Zn 66. Antes da análise, as amostras foram diluídas 10 vezes com ácido nítrico $0,4 \mathrm{~mol} \mathrm{~L}^{-1}$, sendo adicionado ródio como padrão interno. Os resultados da avaliação da exatidão e precisão do método foram realizados com o material de referência Seronorm Level 2 (Tabela 3).

Cromo foi determinado por espectrometria de emissão óptica com fonte de plasma (ICP-OES) no espectrômetro OPTIMA 4300 DV (PerkinElmer, USA), nas condições padrão do instrumento (Tabela 1). Esta técnica foi escolhida para evitar a interferência espectral de ${ }^{40} \mathrm{Ar}^{12} \mathrm{C}$ sobre ${ }^{52} \mathrm{Cr}$ na espectrometria de massa, causada pelas altas concentrações de matéria orgânica nas amostras. A avaliação da exatidão e precisão (Tabela 3 ) foi efetuada com os materiais de referência N202 e N203 (PTA-Waters Sub-Program 91, Austrália).

Sódio foi determinado por espectrometria de emissão em chama no fotômetro NK 2004 (Digimed, São Paulo), com chama ar-butano. Nas determinações de sódio, as amostras foram diluídas de 10 a 100 vezes com água, dependendo da concentração do elemento. Para avaliar a influência da concentração de matéria orgânica sobre a exatidão, uma amostra de suco de uva com $29 \mathrm{mg} \mathrm{L}^{-1}$ de sódio foi fortificada com $2 \mathrm{mg} \mathrm{L}^{-1}$ do analito, tendo sido encontrada uma recuperação média de $92 \%$.

\section{Amostras e reagentes}

Foram analisadas 31 amostras de suco de uva não adoçado, de 20 marcas diferentes, provenientes de 8 estados (siglas entre parênteses): Aurora (RS), Bela Ischia (MG), Big Fruit (SE), Casa da Madeira (RS), Casa de Bento (RS), Curumatan (RJ), Dom Cândido (RS), Imbiara (SC), Jandaia (CE), Maguari (MG), Maravilha (SE), Pérgola (RS), Pindorama (AL), Rossoni (RS), Salton (RS), Santal (SP), Serigy (SE), Sinuelo (RS), Superbom (SC) e Uva Só (RS). Segundo as informações fornecidas pelos fabricantes, 9 amostras são isentas de conservantes. Dentre as outras amostras, 15 foram preservadas com benzoato de sódio (INS 211) em conjunto com metabissulfito de sódio (INS 223), 4 com outras misturas de conservantes contendo sódio, e uma com sorbato de potássio (INS 202) em conjunto com dióxido de enxofre (INS 220). As amostras foram filtradas em membrana de $0,45 \mu \mathrm{m}$ (Millipore, USA). No preparo das soluções foram utilizados reagentes de grau analítico e água desmineralizada (Milli-Q). As soluções de calibração para as técnicas de ICP-MS e ICP-OES foram obtidas por diluição de padrões de grau espectroscópico mono- e multielementares (PerkinElmer, USA) com ácido nítrico $0,4 \mathrm{~mol}$ $\mathrm{L}^{-1}$. As soluções de calibração para a técnica de emissão em chama foram obtidas por diluição de padrão monoelementar Titrisol (Merck, Alemanha) com água. 


\section{RESULTADOS E DISCUSSÃO}

\section{Parâmetros de mérito}

Os parâmetros das curvas analíticas estão resumidos na Tabela 2. Os limites de detecção $\left(\mathrm{LD}=3 \mathrm{~s}_{\mathrm{B}}\right.$ ) foram calculados conforme descrito por Miller e Miller. ${ }^{12}$ A exatidão e precisão do método foram avaliados com três materiais de referência, dos quais dois consistem em amostras de urina (Seronorm Level 1 e Seronorm Level 2, Pharmacia, Noruega) e o outro, de água do mar (CASS-3, National Research Council, Canadá). Os resultados da avaliação, resumidos na Tabela 3, encontram-se de acordo com a incerteza das concentrações certificadas.

\section{Contaminantes inorgânicos}

Os teores máximos dos 8 contaminantes inorgânicos determinados nas amostras de suco de uva (As: 0,016; Cd: 0,010; Cr: 0,060; $\mathrm{Cu}$ : 1,28; Ni: 0,032; Pb: 0,016; Sb: 0,0040 e Zn: 1,44 mg L ${ }^{-1}$; Tabela 4) encontram-se dentro dos Limites Máximos de Tolerância (LMT) estabelecidos pelas normas brasileiras, e atendem também às especificações de outros países para este produto. As concentrações médias de cádmio e antimônio (respectivamente 1,10 e $0,94 \mu \mathrm{g} \mathrm{L}^{-1}$ ) são extremamente

Tabela 2. Parâmetros das curvas analíticas

\begin{tabular}{lccccc}
\hline Elemento & $\begin{array}{c}\mathrm{LD} \\
\left(\mu \mathrm{g} \mathrm{L}^{-1}\right)\end{array}$ & $\begin{array}{c}\text { Faixa } \\
\text { linear } \\
\left(\mu \mathrm{g} \mathrm{\textrm {L } ^ { - 1 } )}\right.\end{array}$ & $\mathrm{a}$ & $\mathrm{b}$ & $\mathrm{r}^{2}$ \\
\hline $\mathrm{As}$ & 0,09 & $0,1-20$ & $2,34 \times 10^{4}$ & 88 & 0,9995 \\
$\mathrm{Cd}$ & 0,1 & $1-200$ & $1,39 \times 10^{6}$ & 44 & 0,9999 \\
$\mathrm{Cr}$ & 0,9 & $10-200$ & 59,7 & $1,2 \times 10^{2}$ & 0,9999 \\
$\mathrm{Cu}$ & 0,05 & $5-200$ & $1,10 \times 10^{7}$ & $3,8 \times 10^{3}$ & 0,9998 \\
$\mathrm{Na}$ & $1 \times 10^{2}$ & $2000-8000$ & 0,0741 & $4,8 \times 10^{2}$ & 0,9907 \\
$\mathrm{Ni}$ & 0,07 & $1-200$ & $1,32 \times 10^{7}$ & $1,9 \times 10^{3}$ & 0,9999 \\
$\mathrm{~Pb}$ & 0,02 & $1-200$ & $1,43 \times 10^{7}$ & $1,6 \times 10^{3}$ & 0,9999 \\
$\mathrm{Sb}$ & 0,004 & $1-200$ & $1,39 \times 10^{7}$ & 47 & 0,9999 \\
$\mathrm{Zn}$ & 0,2 & $5-200$ & $4,82 \times 10^{6}$ & $3,5 \times 10^{3}$ & 1,0000 \\
\hline
\end{tabular}

LD: Limite de detecção; a: coef. angular (cps $\mu \mathrm{g}^{-1} \mathrm{~L}$, exceto sódio em $\mu \mathrm{g}^{-1} \mathrm{~L}$ ); b: coef. linear (cps, exceto sódio, adimensional); r: coef. de determinação.

Tabela 3. Determinação de elementos-traço em materiais de referência

\begin{tabular}{lccc}
\hline Elemento & Material & Este trabalho $^{\text {a }}$ & Valor certificado \\
\hline $\mathrm{As}$ & CASS-3 & $1,2 \pm 0,1$ & $1,09 \pm 0,07^{\mathrm{b}}$ \\
$\mathrm{As}$ & Seronorm-1 & $49,5 \pm 0,3$ & $61(49-73)^{\mathrm{c}}$ \\
$\mathrm{As}$ & Seronorm- & $152 \pm 2$ & $153(122-183)^{\mathrm{c}}$ \\
$\mathrm{Cr}$ & PTA N202 & $0,109 \pm 0,001$ & $0,11 \pm 0,01^{\mathrm{d}}$ \\
$\mathrm{Cr}$ & PTA N203 & $10,77 \pm 0,04$ & $11,00 \pm 0,52^{\mathrm{d}}$ \\
$\mathrm{Cd}$ & Seronorm - 2 & $16,7 \pm 0,3$ & $17,3(13,9-20,8)^{\mathrm{c}}$ \\
$\mathrm{Cu}$ & Seronorm - 2 & $54 \pm 1$ & $51(41-61)^{\mathrm{c}}$ \\
$\mathrm{Ni}$ & Seronorm - 2 & $24,0 \pm 0,5$ & $24,1(19,3-29,0)^{\mathrm{c}}$ \\
$\mathrm{Pb}$ & Seronorm - 2 & $77 \pm 1$ & $68,3(54,6-81,9)^{\mathrm{c}}$ \\
$\mathrm{Sb}$ & Seronorm - 2 & $35 \pm 1$ & $33,3(26,7-40,0)^{\mathrm{c}}$ \\
$\mathrm{Zn}$ & Seronorm - 2 & $(1,02 \pm 0,01) \times 10^{3}$ & $875(700-1050)^{\mathrm{c}}$ \\
\hline $\mathrm{Concen}$
\end{tabular}

Concentrações em $\mu \mathrm{g} \mathrm{L}^{-1}$, exceto $\mathrm{Cr}$ em $\mathrm{mg} \mathrm{L}^{-1}$; a: média e desvio padrão, $\mathrm{n}=3$; $\mathrm{b}$ : média e intervalo de confiança; $\mathrm{c}$ : média e faixa aceitável; d: mediana e faixa do interquartil normalizado.
Tabela 4. Teores de contaminantes inorgânicos em suco de uva

\begin{tabular}{lcccccc}
\hline & $\mathrm{N}$ & $\mathrm{Q}$ & Média & $\mathrm{DP}$ & Valor Máximo & $\mathrm{LMT}$ \\
\hline $\mathrm{As}$ & 25 & 20 & 7,4 & 3,5 & 16 & 500 \\
$\mathrm{Cd}$ & 31 & 21 & 1,10 & 0,62 & 10 & 500 \\
$\mathrm{Cr}$ & 25 & 25 & 21 & 13 & 60 & 100 \\
$\mathrm{Cu}$ & 31 & 31 & 0,45 & 0,35 & 1,28 & 30 \\
$\mathrm{Ni}$ & 31 & 31 & 15,6 & 7,5 & 32 & 3000 \\
$\mathrm{~Pb}$ & 31 & 31 & 6,0 & 4,3 & 16 & 400 \\
$\mathrm{Sb}$ & 25 & 24 & 0,94 & 0,77 & 4,0 & 1000 \\
$\mathrm{Zn}$ & 31 & 31 & 0,66 & 0,31 & 1,44 & 25 \\
\hline $\mathrm{Con}$
\end{tabular}

Concentrações em $\mu \mathrm{g} \mathrm{L}^{-1}$, exceto $\mathrm{Cu}$ e $\mathrm{Zn}$ em $\mathrm{mg} \mathrm{L}^{-1} ; \mathrm{N}: \mathrm{n}^{\circ}$ de amostras; Q: $n^{\circ}$ de amostras com níveis detectáveis do analito; DP: desvio padrão; LMT: Limite Máximo de Tolerância.

reduzidas e situam-se muito abaixo dos valores correspondentes de LMT (respectivamente $500 \mu \mathrm{g} \mathrm{L} \mathrm{L}^{-1} \mathrm{e} 1.000$ ). Os teores médios de arsênio, cromo, níquel e chumbo (respectivamente 7,4 ; 20,6 ; 15,6 e 6,0 $\mu \mathrm{g} \mathrm{L}^{-1}$ ), apesar de ligeiramente maiores, também se encontram muito abaixo dos Limites de Tolerância (respectivamente 500, 100, 3000, e $400 \mu \mathrm{g} \mathrm{L}^{-1}$ ). Concentrações médias mais elevadas foram encontradas para cobre e zinco (respectivamente 0,45 e $0,66 \mathrm{mg} \mathrm{L}^{-1}$ ), porém ainda bem abaixo dos LMT correspondentes (respectivamente 30 e $25 \mathrm{mg} \mathrm{L}^{-1}$ ).

O teor médio de arsênio deste trabalho $\left(7,4 \mu \mathrm{g} \mathrm{L}^{-1}\right)$ é ligeiramente inferior aos valores encontrados por Schoof et al. ${ }^{13} \mathrm{em}$ suco de uva nos Estados Unidos (9,2 $\mu \mathrm{g} \mathrm{kg}^{-1}$ de arsênio inorgânico), e pela Agência Alemã de Proteção ao Consumidor e Segurança Alimentar ${ }^{14}$ em sucos de frutas na Alemanha $\left(11,3 \mu \mathrm{g} \mathrm{kg}^{-1}\right)$. Os teores médios de cádmio, cromo e níquel (respectivamente 1,10; 20,6 e 15,6 $\mu \mathrm{g} \mathrm{L}^{-1}$ ) são similares aos obtidos por Mirlean et $a l .{ }^{6}$ em amostras do estado do Rio Grande do Sul (respectivamente 1,3; 38 e $19 \mu \mathrm{g} \mathrm{L}^{-1}$ ). No entanto, a média de chumbo é sensivelmente menor neste trabalho (6,0 versus $\left.117 \mu \mathrm{g} \mathrm{L}^{-1}\right)$. Por outro lado, a média de zinco $(0,66 \mathrm{mg}$ $\left.\mathrm{L}^{-1}\right)$ é ligeiramente maior que a obtida por Mirlean et al. ${ }^{6}(0,307 \mathrm{mg}$ $\left.\mathrm{L}^{-1}\right)$, porém encontra-se próxima à faixa relatada por Morgano et al. ${ }^{5}$ (0,56-0,61 $\left.\mathrm{mg} \mathrm{L}^{-1}\right)$. O valor médio do zinco no suco de uva brasileiro não é muito superior ao encontrado nos produtos espanhol ${ }^{7}(0,46 \mathrm{mg}$ $\left.\mathrm{L}^{-1}\right)$ e norte-americano ${ }^{8}\left(0,50 \mathrm{mg} \mathrm{kg}^{-1}\right)$.

Em relação ao cobre, a média deste trabalho $\left(0,45 \mathrm{mg} \mathrm{L}^{-1}\right)$ é similar à obtida por Mirlean et al. ${ }^{6}\left(0,53 \mathrm{mg} \mathrm{L}^{-1}\right)$, porém Morgano et al. ${ }^{5}$ encontraram valores muito superiores $\left(3,94-3,80 \mathrm{mg} \mathrm{L}^{-1}\right)$, o que deve ser atribuído a uma peculiaridade das duas amostras analisadas por aqueles pesquisadores. O teor de cobre é ligeiramente menor no suco de uva norte americano ${ }^{8}\left(0,28 \mathrm{mg} \mathrm{L}^{-1}\right)$, mas no produto espanhol ${ }^{7}$ a concentração é extremamente mais baixa $\left(0,063 \mathrm{mg} \mathrm{L}^{-1}\right)$. Os teores de cobre mais elevados encontrados no produto nacional parecem resultar da aplicação de sais de cobre (sulfato de cobre, etc.) como fungicidas nas parreiras de uvas no período próximo à colheita, conforme descrito por Artiguez et al.. ${ }^{15}$ Além do cobre, as concentrações de outros contaminantes no suco de uva podem estar relacionadas com estes fungicidas, pois seu uso continuado pode resultar na acumulação dos contaminantes no solo, de onde podem ser absorvidos pelas plantas. Esta hipótese é respaldada pelos trabalhos de Camoni et al., ${ }^{16}$ que relatam a presença de até $72 \mathrm{mg} \mathrm{kg}^{-1}$ de arsênio e $371 \mathrm{mg} \mathrm{kg}^{-1}$ de chumbo em sais de cobre, e de Mirlean et al., ${ }^{6}$ que encontraram $1.309 \mathrm{mg} \mathrm{kg}^{-1}$ de zinco em sulfato de cobre.

\section{Especiação de arsênio}

Em um estudo recente, Assis ${ }^{17}$ analisou amostras de suco de uva através da técnica de eletroforese capilar acoplada à espectrometria de massa, tendo identificado apenas a presença das espécies inorgânicas arsenito e arsenato. Em concordância com este resultado, 
neste trabalho foi considerada apenas a presença daquelas espécies. A determinação das espécies de arsênio (Tabela 5) mostra que, em média, a concentração de $\mathrm{As}(\mathrm{V})$ é maior que a de $\mathrm{As}(\mathrm{III})$, na razão de 2,8:1,0. No entanto, esta razão varia consideravelmente entre as diversas regiões do país, sendo de 4,3 no Sudeste, 2,9 no Sul e 0,8 no Nordeste. Verifica-se também que a razão média As(V):As(III) no Sudeste é significativamente maior $(\mathrm{p}<0,01)$ que a do Nordeste. Esta informação é relevante, levando-se em conta que o íon arsenito é considerado mais tóxico que o arsenato. ${ }^{6}$ Uma possível explicação para o fenômeno é a utilização, por alguns fabricantes, de compostos redutores para a conservação do suco de uva. Estes compostos, como o metabissulfito de sódio, em quantidades elevadas poderiam contribuir para a diminuição da razão As(V):As(III).

Tabela 5. Especiação de arsênio em suco de uva das regiões Nordeste, Sudeste e Sul do Brasil

\begin{tabular}{|c|c|c|c|c|c|c|c|c|}
\hline & \multicolumn{2}{|c|}{$\begin{array}{c}\text { Nordeste } \\
(\mathrm{N}=6)\end{array}$} & \multicolumn{2}{|c|}{$\begin{array}{l}\text { Sudeste } \\
(\mathrm{N}=8)\end{array}$} & \multicolumn{2}{|c|}{$\begin{array}{c}\text { Sul } \\
(\mathrm{N}=9)\end{array}$} & \multicolumn{2}{|c|}{$\begin{array}{c}\text { Todas Regiões } \\
\quad(\mathrm{N}=23)\end{array}$} \\
\hline & Média & DP & Média & DP & Média & DP & Média & DP \\
\hline $\begin{array}{l}\operatorname{As}(\mathrm{III}) \\
+\mathrm{As}(\mathrm{V})\end{array}$ & 5,5 & 3,7 & 8,3 & 2,6 & 4,3 & 4,7 & 5,9 & 4,1 \\
\hline As(III) & 3,3 & 2,3 & 1,7 & 0,6 & 1,4 & 2,0 & 2,0 & 1,8 \\
\hline $\mathrm{As}(\mathrm{V})$ & 2,2 & 1,5 & 6,7 & 2,4 & 3,3 & 3,0 & 4,2 & 3,1 \\
\hline $\begin{array}{l}\mathrm{As}(\mathrm{V}) \\
/ \mathrm{As}(\mathrm{III})\end{array}$ & 0,8 & 0,5 & 4,3 & 1,9 & 2,9 & 2,2 & 2,8 & 2,2 \\
\hline
\end{tabular}

Concentrações em $\mu \mathrm{g} \mathrm{L}{ }^{-1} ; \mathrm{N}: \mathrm{n}^{\circ}$ de amostras; DP: desvio padrão.

\section{Teores de sódio}

A Tabela 6 apresenta os teores de sódio nas amostras, distribuídos por região. Enquanto a média nacional é de $149 \mathrm{mg} \mathrm{L}^{-1}$, observa-se grande variação regional, com médias de 263, 209 e $46 \mathrm{mg} \mathrm{L}^{-1}$ no Nordeste, Sudeste e Sul, respectivamente. Apenas as amostras da região Sul se aproximam do valor de referência dos Estados Unidos ${ }^{8}$ (30 $\left.\mathrm{mg} \mathrm{kg}^{-1}\right)$. Além disso, os teores de sódio no Sul são significativamente menores $(\mathrm{p}<0,001)$ que nas duas outras regiões pesquisadas. Este fato provavelmente decorre do menor emprego de conservantes contendo sódio, pois 9 (69\% do total) das 13 amostras provenientes do Sul eram isentas de conservantes. A Tabela 7 apresenta as concentrações médias de sódio, em função da presença de conservantes. Verifica-se que o teor médio das amostras contendo conservantes à base de sódio $\left(203 \mathrm{mg} \mathrm{L}^{-1}\right)$ difere significativamente $(\mathrm{p}<0,001)$ do teor nos outros produtos $\left(46 \mathrm{mg} \mathrm{L}^{-1}\right)$. Aparentemente, o dado mais relevante para o consumidor não é a localização geográfica do fabricante, mas o emprego de conservantes contendo sódio no processo de fabricação.

Em relação aos teores de sódio, é necessário mencionar ainda a grande disparidade entre as informações fornecidas pelos fabricantes, e os valores obtidos nas análises. Em 18 amostras (62\% do total), as concentrações dos rótulos diferiram em mais de $50 \%$ dos valores obtidos experimentalmente. Em um dos exemplos mais significativos, o produto apresentava no rótulo uma concentração de 20,2 mg de sódio por porção de $200 \mathrm{~mL}$ (equivalente a $101 \mathrm{mg} \mathrm{L}^{-1}$ ), porém a análise

Tabela 6. Sódio em suco de uva das regiões Nordeste, Sudeste e Sul do Brasil

\begin{tabular}{ccccccccc}
\hline & \multicolumn{2}{c}{$\begin{array}{c}\text { Nordeste } \\
(\mathrm{N}=7)\end{array}$} & \multicolumn{2}{c}{ Sudeste } & \multicolumn{2}{c}{ Sul } & \multicolumn{2}{c}{ Todas Regiões } \\
& Média & DP & Média & DP & Média & DP & Média & DP \\
\hline $\mathrm{Na}$ & 263 & 79 & 209 & 51 & 46 & 29 & 149 & 108 \\
\hline
\end{tabular}

Concentrações em mg L ${ }^{-1}$; N: $n^{\circ}$ de amostras; DP: desvio padrão.
Tabela 7. Sódio em suco de uva, em função da presença de conservantes

\begin{tabular}{lcc}
\hline & Sem conservante* $(\mathrm{N}=10)$ & Com conservante** $(\mathrm{N}=19)$ \\
\hline Média & 46 & 203 \\
DP & 34 & 94 \\
Faixa & $19-110$ & $38-344$ \\
\hline
\end{tabular}

Concentrações em $\mathrm{mg} \mathrm{L}^{-1} ; \mathrm{N}: \mathrm{n}^{\mathrm{o}}$ de amostras; *: inclui 1 amostra com conservante isento de sódio; $* *$ : somente conservantes contendo sódio.

forneceu $344 \mathrm{mg} \mathrm{L}^{-1}$, um valor mais de três vezes maior. Casos como este evidenciam uma deficiência de controle de qualidade do produto, tornando inúteis as informações fornecidas nos rótulos.

Outra questão relevante é que a Agência Nacional de Vigilância Sanitária (ANVISA) recomenda o uso de porções de 100 g ou 100 $\mathrm{mL} .{ }^{10}$ Porém, como esta regra não é obrigatória, cada fabricante escolhe arbitrariamente o tamanho da porção, tornando extremamente difícil para o consumidor comparar produtos similares. Foram encontradas nos rótulos porções variando de 25 até $200 \mathrm{~mL}$. Considerando que massas iguais ou inferiores a $5 \mathrm{mg}$ por porção podem ser representadas no rótulo como "não contém sódio", 7 isto significa que, no caso extremo, o produto que "não contém sódio" pode conter até $200 \mathrm{mg} \mathrm{L}^{-1}$ (5 mg por porção de $25 \mathrm{~mL}$ ) do elemento. Este exemplo demonstra a necessidade de tornar obrigatória regra sobre o tamanho da porção, de forma a facilitar ao consumidor o acesso à informação sobre as reais características do produto.

\section{CONCLUSÕES}

O estudo revela que os teores de antimônio, arsênio, cromo, cobre, níquel, chumbo e zinco no suco de uva consumido no Brasil, se situam muito abaixo dos Limites Máximos de Tolerância estabelecidos. $\mathrm{O}$ arsênio inorgânico, na maioria das amostras, encontra-se como As(V), espécie menos tóxica que o As(III). No entanto, esta proporção varia conforme a região, sendo maior no Nordeste que no Sul do país. Em relação ao sódio, também se verificam variações regionais, o que aparentemente resulta do uso exagerado de conservantes contendo sódio. A concentração média de sódio (149 mg $\left.\mathrm{L}^{-1}\right)$ é muito maior que o valor de referência norte-americano (30 $\mathrm{mg} \mathrm{L}^{-1}$ ). Também foram observadas grandes discrepâncias entre os valores informados nos rótulos e os determinados experimentalmente, indicando a necessidade de que o órgão regulador atue para garantir a exatidão das informações presentes nos rótulos. O estudo propõe ainda que a porção de suco seja padronizada em $100 \mathrm{~mL}$, de forma que o consumidor não precise realizar cálculos para comparar os teores de sódio nos diferentes produtos.

\section{AGRADECIMENTOS}

Aos técnicos A. J. Pereira e M. Dupin pelo valioso auxílio na preparação e análise das amostras.

\section{REFERÊNCIAS}

1. Ministério da Saúde; Port. $n^{\circ} 685$ de 28/8/1998; Poder Executivo. Dec. $n^{o} 55.871$ de 09/04/1965.

2. European Communities; Commission Regulation 466/2001; Off. J. Eur. Commun. 2001, 77, 9.

3. Ministry of Health and Family Welfare of India; http://mohfw.nic.in/pfa. htm, acessada em Junho 2007.

4. Mello, L. M. R.; http://www.cnpuv.embrapa.br, acessada em Junho 2007. 
5. Morgano, M. A.; Queiroz, S. C. N.; Ferreira, M. M. C.; Ciênc. Tecnol. Aliment. 1999, 19, 344.

6. Mirlean, N.; Roisenberg, A.; Chies, J. O.; Bull. Environ. Contam. Toxicol. 2005, 75, 968.

7. Olalla, M.; Fernandez, J.; Cabrera, C.; Navarro, M.; Gimenez, R.; Lopez, M. C.; J. Agric. Food Chem. 2004, 52, 2717.

8. United States Department of Agriculture; http://www.ars.usda.gov/ba/ bhnrc/ndl, acessada em Junho 2007.

9. Cornelis, R., ed.; Handbook of Elemental Speciation, Wiley: Chichester, 2003.

10. Agência Nacional de Vigilância Sanitária; Resolução $R D C n^{\circ} 360$ de 26/12/2003.

11. Stroh, A.; Denoyer, E.; Lu, Q.; Völlkopf, U.; Perkin Elmer-Sciex Appl. Report No. FAR-1; PerkinElmer: Norwalk, 1993.
12. Miller, J. C.; Miller, J. N.; Statistics for Analytical Chemistry, $3^{\text {rd }}$ ed., Ellis Horwood: New York, 1993.

13. Schoof, R. A.; Yost, L. J.; Eickhoff, J.; Crecelius, E. A.; Cragin, D. W.; Meacher, D. M.; Menzel, D. B.; Food Chem. Toxicol. 1999, 37, 839.

14. Bundesamt für Verbraucherschutz und Lebensmittelsicherheit; http:// www.bvl.bund.de/nn_496812/EN/01__Food/food__node.html__ nnn=true, acessada em Junho 2007.

15. Artiguez, M. L.; Grilo, A.; Soria, M. L.; Castro, M.; Repetto, M.; Bull. Environ. Contam. Toxicol. 1990, 45, 711.

16. Camoni, I.; Pontecorvo, D.; Rubbiani, M.; Alimonti, A.; Petrucci, F.; Carol, S.; Acta Chim. Hungarica 1991, 128, 545.

17. Assis, R. A.; Tese de Doutorado, Pontifícia Universidade Católica do Rio de Janeiro, Brasil, 2006. 